Journal of Applied AnALysis

Vol. 14, No. 1 (2008), pp. 131-148

\title{
SECOND ORDER DUALITY IN MULTIOBJECTIVE PROGRAMMING
}

\author{
I. AHMAD and Z. HUSAIN
}

Received November 10, 2006 and, in revised form, November 6, 2007

\begin{abstract}
A nonlinear multiobjective programming problem is considered. Weak, strong and strict converse duality theorems are established under generalized second order $(F, \alpha, \rho, d)$-convexity for second order Mangasarian type and general Mond-Weir type vector duals.
\end{abstract}

\section{INTRODUCTION}

In recent years, there has been an increasing interest in generalizations of convexity in connection with sufficiency and duality in optimization problems. It has been found that only a few properties of convex functions are needed for establishing sufficiency and duality theorems. Using properties needed as definitions of new classes of functions, it is possible to generalize the notion of convexity and to extend the validity of theorems to larger classes of optimization problems. Consequently, several classes of generalized convexity have been introduced. More specifically, the concept of

2000 Mathematics Subject Classification. Primary: 90C29, 90C30, 90C46.

Key words and phrases. Multiobjective programming, second order duality, efficient solution, generalized $(F, \alpha, \rho, d)$-convexity.

The research of second author is supported by the Department of Atomic Energy, Government of India, under the NBHM Post-Doctoral Fellowship Program No. 40/9/2005-R \& D II/2398.

ISSN 1425-6908（C) Heldermann Verlag. 
$(F, \rho)$-convexity was introduced by Preda [16], an extension of $F$-convexity [9] and $\rho$-convexity [17], and he used the concept to obtain some duality results for Wolfe vector dual, Mond-Weir vector dual and general Mond-Weir vector dual to multiobjective programming problem. Gulati and Islam [8] established sufficiency and duality results for multiobjective programming problems under generalized $F$-convexity. Later on, Aghezzaf and Hachimi [1] and Ahmad [2] generalized these results involving generalized $(F, \rho)$ convex functions. For a more comprehensive view of optimality conditions and duality results in multiobjective programming, we refer $[6,7,18]$ and references cited therein.

Mangasarian [13] first formulated the second order dual for a nonlinear programming problem and established duality results under somewhat involved assumptions. Mond [14] reproved second order duality theorems under simpler assumptions than those previously used by Mangasarian [13], and showed that the second order dual has computational advantages over the first order dual. Zhang and Mond [19] extended the class of $(F, \rho)$-convex functions to second order $(F, \rho)$-convex functions and obtained duality results for Mangasarian type, Mond-Weir type and general Mond-Weir type multiobjective dual problems.

A newly introduced concept of generalized convexity, named as $(F, \alpha, \rho, d)$-convexity can be viewed in $[4,10,11]$, while $(F, \alpha, \rho, d)$ pseudoconvexity and $(F, \alpha, \rho, d)$-quasiconvexity can be found in [5]. Recently, Ahmad and Husain [3] introduced the class of generalized second order $(F, \alpha, \rho, d)$-convex functions and discussed duality results for MondWeir type vector dual.

Consider the following nonlinear multiobjective programming problem:

(MP) Minimize $f(x)=\left[f_{1}(x), f_{2}(x), \ldots, f_{k}(x)\right]$ subject to $x \in S=\{x \in X: g(x) \leqq 0\}$,

where $f=\left(f_{1}, f_{2}, \ldots, f_{k}\right): X \mapsto \mathbb{R}^{k}, g=\left(g_{1}, g_{2}, \ldots, g_{m}\right): X \mapsto \mathbb{R}^{m}$ are assumed to be twice differentiable functions over $X$, an open subset of $\mathbb{R}^{n}$.

In this paper, we establish duality theorems under generalized second order $(F, \alpha, \rho, d)$-convexity, for second order Mangasarian type and general Mond-Weir type duals associated with (MP). These results extend the results obtained by Mond and Zhang [15], Zhang and Mond [19] and Ahmad $[2]$.

\section{Notations AND PRELIMINARIES}

Throughout the paper, following convention for vectors $x, y \in \mathbb{R}^{n}$ will be followed: $x \geqq y$ if and only if $x_{i} \geqq y_{i}, i=1,2, \ldots, n ; x \geq y$ if and only if $x \geqq y$ and $x \neq y ; x>y$ if and only if $x_{i}>y_{i}, i=1,2, \ldots, n$. 
Definition 2.1. A point $\bar{x} \in S$ is said to be an efficient solution of the vector minimum problem (MP), if there exists no other $x \in S$ such that

$$
f(x) \leq f(\bar{x}) .
$$

In the sequel, we require the following definitions [3].

Definition 2.2. A functional $F: X \times X \times \mathbb{R}^{n} \mapsto \mathbb{R}$ is said to be sublinear in its third argument, if for all $x, \bar{x} \in X$

(i) $F(x, \bar{x} ; a+b) \leqq F(x, \bar{x} ; a)+F(x, \bar{x} ; b)$, for all $a, b \in \mathbb{R}^{n}$,

(ii) $F(x, \bar{x} ; \beta a)=\beta F(x, \bar{x} ; a)$, for all $\beta \in \mathbb{R}, \beta \geqq 0$, and for all $a \in \mathbb{R}^{n}$.

Let $F$ be sublinear and the scalar function $\phi: X \mapsto \mathbb{R}$ be twice differentiable at $\bar{x} \in X$ and $\rho \in \mathbb{R}$.

Definition 2.3. The function $\phi$ is said to be second order $(F, \alpha, \rho, d)$ convex at $\bar{x}$ on $X$, if for all $x \in X$, there exist vector $p \in \mathbb{R}^{n}$, a real valued function $\alpha: X \times X \mapsto \mathbb{R}_{+} \backslash\{0\}$, and a real valued function $d(\cdot, \cdot): X \times X \mapsto \mathbb{R}$ such that

$$
\begin{aligned}
\phi(x)-\phi(\bar{x})+\frac{1}{2} p^{t} \nabla^{2} \phi(\bar{x}) p \geqq & F\left(x, \bar{x} ; \alpha(x, \bar{x})\left\{\nabla \phi(\bar{x})+\nabla^{2} \phi(\bar{x}) p\right\}\right) \\
& +\rho d^{2}(x, \bar{x}) .
\end{aligned}
$$

If for all $x \in X, x \neq \bar{x}$, the above inequality holds as strict inequality, then $\phi$ is said to be strictly second order $(F, \alpha, \rho, d)$-convex at $\bar{x}$ on $X$.

Definition 2.4. The function $\phi$ is said to be second order $(F, \alpha, \rho, d)$ pseudoconvex at $\bar{x}$ on $X$, if for all $x \in X$, there exist vector $p \in \mathbb{R}^{n}$, a real valued function $\alpha: X \times X \mapsto \mathbb{R}_{+} \backslash\{0\}$, and a real valued function $d(\cdot, \cdot): X \times X \mapsto \mathbb{R}$ such that

$$
\begin{aligned}
\phi(x) & <\phi(\bar{x})-\frac{1}{2} p^{t} \nabla^{2} \phi(\bar{x}) p \Rightarrow F\left(x, \bar{x} ; \alpha(x, \bar{x})\left\{\nabla \phi(\bar{x})+\nabla^{2} \phi(\bar{x}) p\right\}\right) \\
& <-\rho d^{2}(x, \bar{x}) .
\end{aligned}
$$

Definition 2.5. The function $\phi$ is said to be strictly second order $(F, \alpha, \rho, d)$-pseudoconvex at $\bar{x}$ on $X$, if for all $x \in X, x \neq \bar{x}$, there exist vector $p \in \mathbb{R}^{n}$, a real valued function $\alpha: X \times X \mapsto \mathbb{R}_{+} \backslash\{0\}$, and a real valued function $d(\cdot, \cdot): X \times X \mapsto \mathbb{R}$ such that

$$
\begin{aligned}
F\left(x, \bar{x} ; \alpha(x, \bar{x})\left\{\nabla \phi(\bar{x})+\nabla^{2} \phi(\bar{x}) p\right\}\right) & \geqq-\rho d^{2}(x, \bar{x}) \Rightarrow \phi(x) \\
& >\phi(\bar{x})-\frac{1}{2} p^{t} \nabla^{2} \phi(\bar{x}) p,
\end{aligned}
$$


or equivalently

$$
\begin{aligned}
\phi(x) & \leqq \phi(\bar{x})-\frac{1}{2} p^{t} \nabla^{2} \phi(\bar{x}) p \Rightarrow F\left(x, \bar{x} ; \alpha(x, \bar{x})\left\{\nabla \phi(\bar{x})+\nabla^{2} \phi(\bar{x}) p\right\}\right) \\
& <-\rho d^{2}(x, \bar{x}) .
\end{aligned}
$$

Definition 2.6. The function $\phi$ is said to be second order $(F, \alpha, \rho, d)$ quasiconvex at $\bar{x}$ on $X$, if for all $x \in X$, there exist vector $p \in \mathbb{R}^{n}$, a real valued function $\alpha: X \times X \mapsto \mathbb{R}_{+} \backslash\{0\}$, and a real valued function $d(\cdot, \cdot): X \times X \mapsto \mathbb{R}$ such that

$$
\begin{aligned}
\phi(x) & \leqq \phi(\bar{x})-\frac{1}{2} p^{t} \nabla^{2} \phi(\bar{x}) p \Rightarrow F\left(x, \bar{x} ; \alpha(x, \bar{x})\left\{\nabla \phi(\bar{x})+\nabla^{2} \phi(\bar{x}) p\right\}\right) \\
& \leqq-\rho d^{2}(x, \bar{x}),
\end{aligned}
$$

or equivalently

$$
\begin{aligned}
F\left(x, \bar{x} ; \alpha(x, \bar{x})\left\{\nabla \phi(\bar{x})+\nabla^{2} \phi(\bar{x}) p\right\}\right) & >-\rho d^{2}(x, \bar{x}) \Rightarrow \phi(x) \\
& >\phi(\bar{x})-\frac{1}{2} p^{t} \nabla^{2} \phi(\bar{x}) p .
\end{aligned}
$$

A $k$-dimensional vector function $\psi=\left(\psi_{1}, \psi_{2}, \ldots, \psi_{k}\right)$ is said to be second order $(F, \alpha, \rho, d)$-convex, if each $\psi_{i}, i=1,2, \ldots, k$, is second order $\left(F, \alpha, \rho_{i}, d\right)$-convex for the same sublinear functional $F$. Other definitions follow similarly.

Remark 2.1. Let $\alpha(x, \bar{x})=1$. Then second order $(F, \alpha, \rho, d)$-convexity becomes the second order $(F, \rho)$-convexity introduced by Zhang and Mond [19]. In addition, if we set second order term equal to zero i.e., $p=0$, it reduces to $(F, \rho)$-convexity in $[2,16]$.

In [12], Maeda derived the following necessary conditions for a feasible solution $x^{*}$ to be an efficient solution of (MP) under generalized Guignard constraint qualification (GGCQ). We need these conditions in the proof of strong duality theorems.

Theorem 2.1 (Kuhn-Tucker Type Necessary Conditions). Assume that $x^{*}$ is an efficient solution for $(M P)$ at which the generalized Guignard constraint qualification (GGCQ) is satisfied. Then there exist $\lambda^{*} \in \mathbb{R}^{k}$ and $y^{*} \in \mathbb{R}^{m}$, such that

$$
\begin{aligned}
& \lambda^{* t} \nabla f\left(x^{*}\right)+y^{* t} \nabla g\left(x^{*}\right)=0, \\
& y^{* t} g\left(x^{*}\right)=0, \\
& y^{*} \geqq 0, \\
& \lambda^{*}>0, \lambda^{* t} e=1 .
\end{aligned}
$$




\section{MANGASARIAN TYPE SECOND ORDER DUALITY}

In this section, we consider the following Mangasarian type second order dual associated with multiobjective problem (MP) and establish weak, strong and strict converse duality theorems under generalized second order $(F, \alpha, \rho, d)$-convexity:

(WD) Maximize $\left(f_{1}(u)+y^{t} g(u)-\frac{1}{2} p^{t} \nabla^{2}\left[f_{1}(u)+y^{t} g(u)\right] p, \ldots\right.$ ，

subject to

$$
\left.f_{k}(u)+y^{t} g(u)-\frac{1}{2} p^{t} \nabla^{2}\left[f_{k}(u)+y^{t} g(u)\right] p\right)
$$

$$
\begin{aligned}
\nabla \lambda^{t} f(u)+\nabla^{2} \lambda^{t} f(u) p+\nabla y^{t} g(u)+\nabla^{2} y^{t} g(u) p & =0, \\
y & \geqq 0, \\
\lambda & >0, \\
\lambda^{t} e & =1,
\end{aligned}
$$

where $e=(1,1, \ldots, 1) \in \mathbb{R}^{k}, \lambda$ is a $k$-dimensional vector, and is an $m$ dimensional vector.

Theorem 3.1 (Weak Duality). Suppose that for all feasible $x$ in $(M P)$ and all feasible $(u, y, \lambda, p)$ in $(W D)$

(i) $f_{i}, i=1,2, \ldots, k$, is second order $\left(F, \alpha, \rho_{i}, d\right)$-convex at $u$, and $g_{j}$, $j=1,2, \ldots, m$, is second order $\left(F, \alpha, \sigma_{j}, d\right)$-convex at $u$, and

$$
\frac{1}{\alpha(x, u)}\left(\sum_{i=1}^{k} \lambda_{i} \rho_{i}+\sum_{j=1}^{m} \sigma_{j} y_{j}\right) \geqq 0 ;
$$

or

(ii) $\lambda^{t} f+y^{t} g$ is second order $(F, \alpha, \rho, d)$-pseudoconvex at $u$, and

$$
\frac{\rho}{\alpha(x, u)} \geqq 0 .
$$

Then, the following cannot hold

$$
f_{i}(x) \leqq f_{i}(u)+y^{t} g(u)-\frac{1}{2} p^{t} \nabla^{2}\left[f_{i}(u)+y^{t} g(u)\right] p, \text { for all } i \in K,
$$

and

$$
f_{j}(x)<f_{j}(u)+y^{t} g(u)-\frac{1}{2} p^{t} \nabla^{2}\left[f_{j}(u)+y^{t} g(u)\right] p, \text { for some } j \in K \text {. }
$$


Proof. Let $x$ be feasible for (MP) and $(u, y, \lambda, p)$ feasible for (WD). Suppose contrary to the result that (3.5) and (3.6) hold. By $y \geqq 0$ and $g(x) \leqq 0$, we have

$$
\begin{array}{r}
f_{i}(x)+y^{t} g(x) \leqq f_{i}(u)+y^{t} g(u)-\frac{1}{2} p^{t} \nabla^{2}\left[f_{i}(u)+y^{t} g(u)\right] p, \\
\text { for all } i \in K,
\end{array}
$$

and

$$
\begin{array}{r}
f_{j}(x)+y^{t} g(x)<f_{j}(u)+y^{t} g(u)-\frac{1}{2} p^{t} \nabla^{2}\left[f_{j}(u)+y^{t} g(u)\right] p, \\
\quad \text { for some } j \in K .
\end{array}
$$

(i) In view of the hypothesis $\lambda>0$ and $\lambda^{t} e=1$, we get

$$
\lambda^{t} f(x)+y^{t} g(x)<\lambda^{t} f(u)+y^{t} g(u)-\frac{1}{2} p^{t} \nabla^{2}\left[\lambda^{t} f(u)+y^{t} g(u)\right] p .
$$

The second order $\left(F, \alpha, \rho_{i}, d\right)$-convexity of $f_{i}, i=1,2, \ldots, k$, and the second order $\left(F, \alpha, \sigma_{j}, d\right)$-convexity of $g_{j}, j=1,2, \ldots, m$, at $u$ imply

$$
\begin{aligned}
& f_{i}(x)-f_{i}(u)+\frac{1}{2} p^{t} \nabla^{2} f_{i}(u) p \\
& \geqq F\left(x, u ; \alpha(x, u)\left\{\nabla f_{i}(u)+\nabla^{2} f_{i}(u) p\right\}\right)+\rho_{i} d^{2}(x, u),
\end{aligned}
$$

$i=1,2, \ldots, k$, and

$$
\begin{aligned}
& g_{j}(x)-g_{j}(u)+\frac{1}{2} p^{t} \nabla^{2} g_{j}(u) p \\
& \geqq F\left(x, u ; \alpha(x, u)\left\{\nabla g_{j}(u)+\nabla^{2} g_{j}(u) p\right\}\right)+\sigma_{j} d^{2}(x, u),
\end{aligned}
$$

$j=1,2, \ldots, m$.

On multiplying the first inequality by $\lambda_{i}>0$ and second by $y_{j} \geqq 0$, and then summing up to get

$$
\begin{aligned}
& \lambda^{t} f(x)+y^{t} g(x)-\lambda^{t} f(u)-y^{t} g(u)+\frac{1}{2} p^{t} \nabla^{2}\left[\lambda^{t} f(u)+y^{t} g(u)\right] p \\
& \geqq F\left(x, u ; \alpha(x, u)\left\{\nabla \lambda^{t} f(u)+\nabla^{2} \lambda^{t} f(u) p\right\}\right) \\
& +F\left(x, u ; \alpha(x, u)\left\{\nabla y^{t} g(u)+\nabla^{2} y^{t} g(u) p\right\}\right) \\
& +\left(\sum_{i=1}^{k} \lambda_{i} \rho_{i}+\sum_{j=1}^{m} \sigma_{j} y_{j}\right) d^{2}(x, u),
\end{aligned}
$$

which in view of (3.9) and the sublinearity of $F$ with $\alpha(x, u)>0$ gives

$$
\begin{aligned}
& F\left(x, u ; \nabla \lambda^{t} f(u)+\nabla^{2} \lambda^{t} f(u) p+\nabla y^{t} g(u)+\nabla^{2} y^{t} g(u) p\right) \\
& <-\frac{1}{\alpha(x, u)}\left(\sum_{i=1}^{k} \lambda_{i} \rho_{i}+\sum_{j=1}^{m} \sigma_{j} y_{j}\right) d^{2}(x, u) .
\end{aligned}
$$


Since

$$
\frac{1}{\alpha(x, u)}\left(\sum_{i=1}^{k} \lambda_{i} \rho_{i}+\sum_{j=1}^{m} \sigma_{j} y_{j}\right) \geqq 0,
$$

the above inequality implies

$$
F\left(x, u ; \nabla \lambda^{t} f(u)+\nabla^{2} \lambda^{t} f(u) p+\nabla y^{t} g(u)+\nabla^{2} y^{t} g(u) p\right)<0,
$$

a contradiction to $(3.1)$, since $F(x, u ; 0)=0$.

(ii) The second order $(F, \alpha, \rho, d)$-pseudoconvexity of $\lambda^{t} f+y^{t} g$ at $u$ along with (3.9) yields

$$
\begin{aligned}
& F\left(x, u ; \alpha(x, u)\left\{\nabla \lambda^{t} f(u)+\nabla^{2} \lambda^{t} f(u) p+\nabla y^{t} g(u)+\nabla^{2} y^{t} g(u) p\right\}\right) \\
& <-\rho d^{2}(x, u),
\end{aligned}
$$

which together with the sublinearity of $F$ and $\alpha(x, u)>0$ gives

$$
\begin{aligned}
& F\left(x, u ; \nabla \lambda^{t} f(u)+\nabla^{2} \lambda^{t} f(u) p+\nabla y^{t} g(u)+\nabla^{2} y^{t} g(u) p\right) \\
& <-\frac{\rho}{\alpha(x, u)} d^{2}(x, u) .
\end{aligned}
$$

Since

then we have

$$
\frac{\rho}{\alpha(x, u)} \geqq 0
$$

$$
F\left(x, u ; \nabla \lambda^{t} f(u)+\nabla^{2} \lambda^{t} f(u) p+\nabla y^{t} g(u)+\nabla^{2} y^{t} g(u) p\right)<0,
$$

which again contradicts $(3.1)$, since $F(x, u ; 0)=0$.

Theorem 3.2 (Strong Duality). Let $\bar{x}$ be an efficient solution of $(M P)$ at which the generalized Guignard constraint qualification $(G G C Q)$ is satisfied. Then there exist $\bar{y} \in \mathbb{R}^{m}$ and $\bar{\lambda} \in \mathbb{R}^{k}$, such that $(\bar{x}, \bar{y}, \bar{\lambda}, \bar{p}=0)$ is feasible for $(W D)$ and the corresponding objective values of $(M P)$ and $(W D)$ are equal.

If, in addition, the assumptions of weak duality (Theorem 3.1) hold for all feasible solutions of (MP) and (WD), then $(\bar{x}, \bar{y}, \bar{\lambda}, \bar{p}=0)$ is an efficient solution of (WD).

Proof. Since $\bar{x}$ is an efficient solution of (MP) at which the generalized Guignard constraint qualification (GGCQ) is satisfied, then by Theorem 2.1 , there exist $\bar{y} \in \mathbb{R}^{m}$ and $\bar{\lambda} \in \mathbb{R}^{k}$, such that

$$
\begin{aligned}
& \bar{\lambda}^{t} \nabla f(\bar{x})+\bar{y}^{t} \nabla g(\bar{x})=0, \\
& \bar{y}^{t} g(\bar{x})=0, \\
& \bar{y} \geqq 0,
\end{aligned}
$$




$$
\bar{\lambda}>0, \bar{\lambda}^{t} e=1 \text {. }
$$

Therefore, $(\bar{x}, \bar{y}, \bar{\lambda}, \bar{p}=0)$ is feasible for (WD) and the corresponding objective values of (MP) and (WD) are equal. The efficiency of this feasible solution for (WD) thus follows from weak duality (Theorem 3.1).

Theorem 3.3 (Strict Converse Duality). Let $\bar{x}$ and $(\bar{u}, \bar{y}, \bar{\lambda}, \bar{p})$ be the efficient solutions of $(M P)$ and $(W D)$ respectively, such that

$$
\bar{\lambda}^{t} f(\bar{x})=\bar{\lambda}^{t} f(\bar{u})+\bar{y}^{t} g(\bar{u})-\frac{1}{2} \bar{p}^{t} \nabla^{2}\left[\bar{\lambda}^{t} f(\bar{u})+\bar{y}^{t} g(\bar{u})\right] \bar{p} .
$$

Suppose that $f_{i}, i=1,2, \ldots, k$, is strictly second order $\left(F, \alpha, \rho_{i}, d\right)$-convex at $\bar{u}$, and $g_{j}, j=1,2, \ldots, m$, is second $\operatorname{order}\left(F, \alpha, \sigma_{j}, d\right)$-convex at $\bar{u}$, and

$$
\frac{1}{\alpha(\bar{x}, \bar{u})}\left(\sum_{i=1}^{k} \bar{\lambda}_{i} \rho_{i}+\sum_{j=1}^{m} \sigma_{j} \bar{y}_{j}\right) \geqq 0 .
$$

Then $\bar{x}=\bar{u}$; that is, $\bar{u}$ is an efficient solution of $(M P)$.

Proof. We assume that $\bar{x} \neq \bar{u}$ and exhibit a contradiction. Since $f_{i}, i=$ $1,2, \ldots, k$, is strictly second order $\left(F, \alpha, \rho_{i}, d\right)$-convex at $\bar{u}$, and $g_{j}, j=$ $1,2, \ldots, m$, is second order $\left(F, \alpha, \sigma_{j}, d\right)$-convex at $\bar{u}$, we have

$$
\begin{aligned}
& f_{i}(\bar{x})-f_{i}(\bar{u})+\frac{1}{2} \bar{p}^{t} \nabla^{2} f_{i}(\bar{u}) \bar{p} \\
& >F\left(\bar{x}, \bar{u} ; \alpha(\bar{x}, \bar{u})\left\{\nabla f_{i}(\bar{u})+\nabla^{2} f_{i}(\bar{u}) \bar{p}\right\}\right)+\rho_{i} d^{2}(\bar{x}, \bar{u}),
\end{aligned}
$$

$i=1,2, \ldots, k$, and

$$
\begin{aligned}
& g_{j}(\bar{x})-g_{j}(\bar{u})+\frac{1}{2} \bar{p}^{t} \nabla^{2} g_{j}(\bar{u}) \bar{p} \\
& \geqq F\left(\bar{x}, \bar{u} ; \alpha(\bar{x}, \bar{u})\left\{\nabla g_{j}(\bar{u})+\nabla^{2} g_{j}(\bar{u}) \bar{p}\right\}\right)+\sigma_{j} d^{2}(\bar{x}, \bar{u}),
\end{aligned}
$$

$j=1,2, \ldots, m$.

On multiplying the first inequality by $\bar{\lambda}_{i}>0$ and second by $\bar{y}_{j} \geqq 0$ and then summing up to get

$$
\begin{aligned}
& \bar{\lambda}^{t} f(\bar{x})+\bar{y}^{t} g(\bar{x})-\bar{\lambda}^{t} f(\bar{u})-\bar{y}^{t} g(\bar{u})+\frac{1}{2} \bar{p}^{t} \nabla^{2}\left[\bar{\lambda}^{t} f(\bar{u})+\bar{y}^{t} g(\bar{u})\right] \bar{p} \\
& >F\left(\bar{x}, \bar{u} ; \alpha(\bar{x}, \bar{u})\left\{\nabla \bar{\lambda}^{t} f(\bar{u})+\nabla^{2} \bar{\lambda}^{t} f(\bar{u}) \bar{p}\right\}\right) \\
& +F\left(\bar{x}, \bar{u} ; \alpha(\bar{x}, \bar{u})\left\{\nabla \bar{y}^{t} g(\bar{u})+\nabla^{2} \bar{y}^{t} g(\bar{u}) \bar{p}\right\}\right) \\
& +\left(\sum_{i=1}^{k} \bar{\lambda}_{i} \rho_{i}+\sum_{j=1}^{m} \sigma_{j} \bar{y}_{j}\right) d^{2}(\bar{x}, \bar{u}),
\end{aligned}
$$


which in view of (3.10) and the feasibility of $\bar{x}$ for (MP) implies

$$
\begin{aligned}
& F\left(\bar{x}, \bar{u} ; \alpha(\bar{x}, \bar{u})\left\{\nabla \bar{\lambda}^{t} f(\bar{u})+\nabla^{2} \bar{\lambda}^{t} f(\bar{u}) \bar{p}\right\}\right) \\
& +F\left(\bar{x}, \bar{u} ; \alpha(\bar{x}, \bar{u})\left\{\nabla \bar{y}^{t} g(\bar{u})+\nabla^{2} \bar{y}^{t} g(\bar{u}) \bar{p}\right\}\right) \\
& <-\left(\sum_{i=1}^{k} \bar{\lambda}_{i} \rho_{i}+\sum_{j=1}^{m} \sigma_{j} \bar{y}_{j}\right) d^{2}(\bar{x}, \bar{u}) .
\end{aligned}
$$

Since $F$ is sublinear and $\alpha(\bar{x}, \bar{u})>0$, then

$$
\begin{aligned}
& F\left(\bar{x}, \bar{u} ; \nabla \bar{\lambda}^{t} f(\bar{u})+\nabla^{2} \bar{\lambda}^{t} f(\bar{u}) \bar{p}+\nabla \bar{y}^{t} g(\bar{u})+\nabla^{2} \bar{y}^{t} g(\bar{u}) \bar{p}\right) \\
& <-\frac{1}{\alpha(\bar{x}, \bar{u})}\left(\sum_{i=1}^{k} \bar{\lambda}_{i} \rho_{i}+\sum_{j=1}^{m} \sigma_{j} \bar{y}_{j}\right) d^{2}(\bar{x}, \bar{u}),
\end{aligned}
$$

which in view of

$$
\frac{1}{\alpha(\bar{x}, \bar{u})}\left(\sum_{i=1}^{k} \bar{\lambda}_{i} \rho_{i}+\sum_{j=1}^{m} \sigma_{j} \bar{y}_{j}\right) \geqq 0
$$

yields

$$
F\left(\bar{x}, \bar{u} ; \nabla \bar{\lambda}^{t} f(\bar{u})+\nabla^{2} \bar{\lambda}^{t} f(\bar{u}) \bar{p}+\nabla \bar{y}^{t} g(\bar{u})+\nabla^{2} \bar{y}^{t} g(\bar{u}) \bar{p}\right)<0,
$$

a contradiction to $(3.1)$, since $F(\bar{x}, \bar{u} ; 0)=0$. Hence, $\bar{x}=\bar{u}$.

\section{General Mond-Weir type Second order Duality}

In this section, we consider the following general Mond-Weir type second order dual associated with multiobjective problem (MP):

(GMD) Maximize $\left(f_{1}(u)+\sum_{i \in I_{0}} y_{i} g_{i}(u)-\frac{1}{2} p^{t} \nabla^{2}\left[f_{1}(u)+\sum_{i \in I_{0}} y_{i} g_{i}(u)\right] p\right.$,
$\left.\ldots, f_{k}(u)+\sum_{i \in I_{0}} y_{i} g_{i}(u)-\frac{1}{2} p^{t} \nabla^{2}\left[f_{k}(u)+\sum_{i \in I_{0}} y_{i} g_{i}(u)\right] p\right)$

subject to

$$
\begin{array}{r}
\nabla \lambda^{t} f(u)+\nabla^{2} \lambda^{t} f(u) p+\nabla y^{t} g(u)+\nabla^{2} y^{t} g(u) p=0, \\
\sum_{i \in I_{\beta}} y_{i} g_{i}(u)-\frac{1}{2} p^{t} \nabla^{2} \sum_{i \in I_{\beta}} y_{i} g_{i}(u) p \geqq 0, \beta=1,2, \ldots, r, \\
y \geqq 0, \\
\lambda>0, \\
\lambda^{t} e=1,
\end{array}
$$


where $I_{\beta} \subseteq M=\{1,2, \ldots, m\}, \beta=0,1,2, \ldots, r$, with $I_{\beta} \cap I_{\gamma}=\emptyset$ if $\beta \neq \gamma$ and $\bigcup_{\beta=0}^{r} I_{\beta}=M$.

Theorem 4.1 (Weak Duality). Suppose that for all feasible $x$ in $(M P)$ and all feasible $(u, y, \lambda, p)$ in $(G M D)$

(i) $\sum_{i \in I_{\beta}} y_{i} g_{i}, \beta=1,2, \ldots, r$, is second $\operatorname{order}\left(F, \alpha, \sigma_{\beta}, d\right)$-quasiconvex at $u$, and assume that any one of the following conditions holds:

(ii) $I_{0} \neq M$, for all $i \in K, f_{i}+\sum_{i \in I_{0}} y_{i} g_{i}$ is second order $\left(F, \alpha_{1}, \rho_{i}, d\right)$ quasiconvex and for some $j \in K, f_{j}+\sum_{i \in I_{0}} y_{i} g_{i}$ is second order $\left(F, \alpha_{1}, \rho_{j}, d\right)$-pseudoconvex at $u$, and

$$
\left[\frac{1}{\alpha(x, u)} \sum_{\beta=1}^{r} \sigma_{\beta}+\frac{1}{\alpha_{1}(x, u)} \sum_{i=1}^{k} \lambda_{i} \rho_{i}\right] \geqq 0 ;
$$

(iii) $I_{0} \neq M, \lambda^{t} f+\sum_{i \in I_{0}} y_{i} g_{i}$ is second order $\left(F, \alpha_{2}, \rho, d\right)$-pseudoconvex at $u$, and

$$
\left[\frac{1}{\alpha(x, u)} \sum_{\beta=1}^{r} \sigma_{\beta}+\frac{\rho}{\alpha_{2}(x, u)}\right] \geqq 0 .
$$

Then, the following cannot hold

$$
f_{i}(x) \leqq f_{i}(u)+\sum_{i \in I_{0}} y_{i} g_{i}(u)-\frac{1}{2} p^{t} \nabla^{2}\left[f_{i}(u)+\sum_{i \in I_{0}} y_{i} g_{i}(u)\right] p
$$

and

$$
f_{j}(x)<f_{j}(u)+\sum_{i \in I_{0}} y_{i} g_{i}(u)-\frac{1}{2} p^{t} \nabla^{2}\left[f_{j}(u)+\sum_{i \in I_{0}} y_{i} g_{i}(u)\right] p
$$

Proof. (i) Let $x$ be any feasible solution in (MP) and $(u, y, \lambda, p)$ be any feasible solution in (GMD). Then $y \geqq 0, g(x) \leqq 0$ and (4.2) yields

$$
\begin{aligned}
& \sum_{i \in I_{\beta}} y_{i} g_{i}(x) \leqq 0 \leqq \sum_{i \in I_{\beta}} y_{i} g_{i}(u)-\frac{1}{2} p^{t} \nabla^{2} \sum_{i \in I_{\beta}} y_{i} g_{i}(u) p, \\
& \beta=1,2, \ldots, r \text {. }
\end{aligned}
$$


Since $\sum_{i \in I_{\beta}} y_{i} g_{i}, \beta=1,2, \ldots, r$, is second order $\left(F, \alpha, \sigma_{\beta}, d\right)$-quasiconvex at $u$, then (4.8) gives

$$
\begin{aligned}
& F\left(x, u ; \alpha(x, u)\left\{\nabla \sum_{i \in I_{\beta}} y_{i} g_{i}(u)+\nabla^{2} \sum_{i \in I_{\beta}} y_{i} g_{i}(u) p\right\}\right) \leqq-\sigma_{\beta} d^{2}(x, u), \\
& \beta=1,2, \ldots, r .
\end{aligned}
$$

The sublinearity of $F$ with $\alpha(x, u)>0$ implies

$$
\begin{aligned}
& F\left(x, u ; \nabla \sum_{i \in M \backslash I_{0}} y_{i} g_{i}(u)+\nabla^{2} \sum_{i \in M \backslash I_{0}} y_{i} g_{i}(u) p\right) \\
& \leqq \sum_{\beta=1}^{r} F\left(x, u ; \nabla \sum_{i \in I_{\beta}} y_{i} g_{i}(u)+\nabla^{2} \sum_{i \in I_{\beta}} y_{i} g_{i}(u) p\right) \\
& \leqq-\frac{1}{\alpha(x, u)}\left(\sum_{\beta=1}^{r} \sigma_{\beta}\right) d^{2}(x, u) .
\end{aligned}
$$

Now suppose contrary to the result that (4.6) and (4.7) hold. By $y \geqq 0$ and $g(x) \leqq 0$, it follows that

$$
f_{i}(x)+\sum_{i \in I_{0}} y_{i} g_{i}(x) \leqq f_{i}(u)+\sum_{i \in I_{0}} y_{i} g_{i}(u)-\frac{1}{2} p^{t} \nabla^{2}\left[f_{i}(u)+\sum_{i \in I_{0}} y_{i} g_{i}(u)\right] p
$$

and

$$
\begin{aligned}
f_{j}(x)+\sum_{i \in I_{0}} y_{i} g_{i}(x)<f_{j}(u)+\sum_{i \in I_{0}} y_{i} g_{i}(u)-\frac{1}{2} p^{t} \nabla^{2} & {\left[f_{j}(u)+\sum_{i \in I_{0}} y_{i} g_{i}(u)\right] p } \\
& \text { for some } j \in K .
\end{aligned}
$$

(ii) Using the second order $\left(F, \alpha_{1}, \rho_{i}, d\right)$-quasiconvexity of $f_{i}+\sum_{i \in I_{0}} y_{i} g_{i}$, for all $i \in K$, and the second order $\left(F, \alpha_{1}, \rho_{j}, d\right)$-pseudoconvexity of $f_{j}+\sum_{i \in I_{0}} y_{i} g_{i}$, for some $j \in K$, we have from (4.10) and (4.11)

$$
\begin{aligned}
& F\left(x, u ; \alpha_{1}(x, u)\left\{\nabla f_{i}(u)+\nabla^{2} f_{i}(u) p+\nabla \sum_{i \in I_{0}} y_{i} g_{i}(u)+\nabla^{2} \sum_{i \in I_{0}} y_{i} g_{i}(u) p\right\}\right) \\
& \leqq-\rho_{i} d^{2}(x, u),
\end{aligned}
$$


for all $i \in K$, and

$$
\begin{aligned}
& F\left(x, u ; \alpha_{1}(x, u)\left\{\nabla f_{j}(u)+\nabla^{2} f_{j}(u) p+\nabla \sum_{i \in I_{0}} y_{i} g_{i}(u)+\nabla^{2} \sum_{i \in I_{0}} y_{i} g_{i}(u) p\right\}\right) \\
& <-\rho_{j} d^{2}(x, u),
\end{aligned}
$$

for some $j \in K$.

The sublinearity of $F, \alpha_{1}(x, u)>0, \lambda>0$ and $\lambda^{t} e=1$ imply

$$
\begin{aligned}
& F\left(x, u ; \nabla \lambda^{t} f(u)+\nabla^{2} \lambda^{t} f(u) p+\nabla \sum_{i \in I_{0}} y_{i} g_{i}(u)+\nabla^{2} \sum_{i \in I_{0}} y_{i} g_{i}(u) p\right) \\
& <-\frac{1}{\alpha_{1}(x, u)}\left(\sum_{i=1}^{k} \lambda_{i} \rho_{i}\right) d^{2}(x, u) .
\end{aligned}
$$

Using (4.9), (4.12) and the sublinearity of $F$, we get

$$
\begin{aligned}
& F\left(x, u ; \nabla \lambda^{t} f(u)+\nabla^{2} \lambda^{t} f(u) p+\nabla y^{t} g(u)+\nabla^{2} y^{t} g(u) p\right) \\
& \leqq F\left(x, u ; \nabla \lambda^{t} f(u)+\nabla^{2} \lambda^{t} f(u) p+\nabla \sum_{i \in I_{0}} y_{i} g_{i}(u)+\nabla^{2} \sum_{i \in I_{0}} y_{i} g_{i}(u) p\right) \\
& +F\left(x, u ; \nabla \sum_{i \in M \backslash I_{0}} y_{i} g_{i}(u)+\nabla^{2} \sum_{i \in M \backslash I_{0}} y_{i} g_{i}(u) p\right) \\
& <-\left[\frac{1}{\alpha(x, u)} \sum_{\beta=1}^{r} \sigma_{\beta}+\frac{1}{\alpha_{1}(x, u)} \sum_{i=1}^{k} \lambda_{i} \rho_{i}\right] d^{2}(x, u) \\
& \leqq 0 \quad\left[\text { since }\left[\frac{1}{\alpha(x, u)} \sum_{\beta=1}^{r} \sigma_{\beta}+\frac{1}{\alpha_{1}(x, u)} \sum_{i=1}^{k} \lambda_{i} \rho_{i}\right] \geqq 0\right),
\end{aligned}
$$

which is a contradiction to $(4.1)$, since $F(x, u ; 0)=0$.

(iii) By $\lambda>0$ and $\lambda^{t} e=1$, (4.10) and (4.11) imply

$\lambda^{t} f(x)+\sum_{i \in I_{0}} y_{i} g_{i}(x)<\lambda^{t} f(u)+\sum_{i \in I_{0}} y_{i} g_{i}(u)-\frac{1}{2} p^{t} \nabla^{2}\left[\lambda^{t} f(u)+\sum_{i \in I_{0}} y_{i} g_{i}(u)\right] p$,

which by the second order $\left(F, \alpha_{2}, \rho, d\right)$-pseudoconvexity of $\lambda^{t} f+\sum_{i \in I_{0}} y_{i} g_{i}$ at $u$ gives 


$$
\begin{aligned}
& F\left(x, u ; \alpha_{2}(x, u)\left\{\nabla \lambda^{t} f(u)+\nabla^{2} \lambda^{t} f(u) p+\nabla \sum_{i \in I_{0}} y_{i} g_{i}(u)+\nabla^{2} \sum_{i \in I_{0}} y_{i} g_{i}(u) p\right\}\right) \\
& <-\rho d^{2}(x, u) .
\end{aligned}
$$

Using (4.9), (4.13) and the sublinearity of $F$ with $\alpha_{2}(x, u)>0$, we get

$$
\begin{aligned}
& F\left(x, u ; \nabla \lambda^{t} f(u)+\nabla^{2} \lambda^{t} f(u) p+\nabla y^{t} g(u)+\nabla^{2} y^{t} g(u) p\right) \\
& \leqq F\left(x, u ; \nabla \lambda^{t} f(u)+\nabla^{2} \lambda^{t} f(u) p+\nabla \sum_{i \in I_{0}} y_{i} g_{i}(u)+\nabla^{2} \sum_{i \in I_{0}} y_{i} g_{i}(u) p\right) \\
& +F\left(x, u ; \nabla \sum_{i \in M \backslash I_{0}} y_{i} g_{i}(u)+\nabla^{2} \sum_{i \in M \backslash I_{0}} y_{i} g_{i}(u) p\right) \\
& <-\left[\frac{1}{\alpha(x, u)} \sum_{\beta=1}^{r} \sigma_{\beta}+\frac{\rho}{\alpha_{2}(x, u)}\right] d^{2}(x, u) .
\end{aligned}
$$

Since

$$
\left[\frac{1}{\alpha(x, u)} \sum_{\beta=1}^{r} \sigma_{\beta}+\frac{\rho}{\alpha_{2}(x, u)}\right] \geqq 0,
$$

we have

$$
F\left(x, u ; \nabla \lambda^{t} f(u)+\nabla^{2} \lambda^{t} f(u) p+\nabla y^{t} g(u)+\nabla^{2} y^{t} g(u) p\right)<0,
$$

again a contradiction to $(4.1)$, since $F(x, u ; 0)=0$.

We now merely state the following strong duality theorem as its proof would run analogously to that of Theorem 3.2 .

Theorem 4.2 (Strong Duality). Let $\bar{x}$ be an efficient solution of $(M P)$ at which the generalized Guignard constraint qualification $(G G C Q)$ is satisfied. Then there exist $\bar{y} \in \mathbb{R}^{m}$ and $\bar{\lambda} \in \mathbb{R}^{k}$, such that $(\bar{x}, \bar{y}, \bar{\lambda}, \bar{p}=0)$ is feasible for $(G M D)$ and the corresponding objective values of $(M P)$ and $(G M D)$ are equal.

If, in addition, the assumptions of weak duality (Theorem 4.1) hold for all feasible solutions of (MP) and (GMD), then $(\bar{x}, \bar{y}, \bar{\lambda}, \bar{p}=0)$ is an efficient solution of (GMD).

Theorem 4.3 (Strict Converse Duality). Let $\bar{x}$ and $(\bar{u}, \bar{y}, \bar{\lambda}, \bar{p})$ be the efficient solutions of $(M P)$ and $(G M D)$ respectively, such that 


$$
\begin{aligned}
\bar{\lambda}^{t} f(\bar{x})= & \bar{\lambda}^{t} f(\bar{u})+\sum_{i \in I_{0}} \bar{y}_{i} g_{i}(\bar{u}) \\
& -\frac{1}{2} \bar{p}^{t} \nabla^{2}\left[\bar{\lambda}^{t} f(\bar{u})+\sum_{i \in I_{0}} \bar{y}_{i} g_{i}(\bar{u})\right] \bar{p} .
\end{aligned}
$$

Suppose that any one of the following conditions is satisfied:

(i) $I_{0} \neq M, \sum_{i \in I_{\beta}} \bar{y}_{i} g_{i}, \beta=1,2, \ldots, r$ is second order $\left(F, \alpha, \sigma_{\beta}, d\right)$ quasiconvex at $\bar{u}$ and $\bar{\lambda}^{t} f+\sum_{i \in I_{0}} \bar{y}_{i} g_{i}$ is strictly second order $\left(F, \alpha_{1}, \rho, d\right)$-pseudoconvex at $\bar{u}$, and

$$
\left[\frac{1}{\alpha(\bar{x}, \bar{u})} \sum_{\beta=1}^{r} \sigma_{\beta}+\frac{\rho}{\alpha_{1}(\bar{x}, \bar{u})}\right] \geqq 0 ;
$$

(ii) $I_{0} \neq M, \sum_{i \in I_{\beta}} \bar{y}_{i} g_{i}, \beta=1,2, \ldots, r$, is strictly second order $\left(F, \alpha, \sigma_{\beta}, d\right)$ pseudoconvex at $\bar{u}$ and $\bar{\lambda}^{t} f+\sum_{i \in I_{0}} \bar{y}_{i} g_{i}$ is second order $\left(F, \alpha_{1}, \rho, d\right)$ quasiconvex at $\bar{u}$, and

$$
\left[\frac{1}{\alpha(\bar{x}, \bar{u})} \sum_{\beta=1}^{r} \sigma_{\beta}+\frac{\rho}{\alpha_{1}(\bar{x}, \bar{u})}\right] \geqq 0 .
$$

Then $\bar{x}=\bar{u}$; that is, $\bar{u}$ is an efficient solution of $(M P)$.

Proof. We assume that $\bar{x} \neq \bar{u}$ and exhibit a contradiction. Let $\bar{x}$ be feasible for $(\mathrm{MP})$ and $(\bar{u}, \bar{y}, \bar{\lambda}, \bar{p})$ be feasible for (GMD). Then $\bar{y} \geqq 0, g(\bar{x}) \leqq 0$ and (4.2) yields

$$
\begin{aligned}
& \sum_{i \in I_{\beta}} \bar{y}_{i} g_{i}(\bar{x}) \leqq 0 \leqq \sum_{i \in I_{\beta}} \bar{y}_{i} g_{i}(\bar{u})-\frac{1}{2} \bar{p}^{t} \nabla^{2} \sum_{i \in I_{\beta}} \bar{y}_{i} g_{i}(\bar{u}) \bar{p}, \\
& \beta=1,2, \ldots, r,
\end{aligned}
$$

which by the second order $\left(F, \alpha, \sigma_{\beta}, d\right)$-quasiconvexity of $\sum_{i \in I_{\beta}} \bar{y}_{i} g_{i}$ at $\bar{u}$ gives

$$
F\left(\bar{x}, \bar{u} ; \alpha(\bar{x}, \bar{u})\left\{\nabla \sum_{i \in I_{\beta}} \bar{y}_{i} g_{i}(\bar{u})+\nabla^{2} \sum_{i \in I_{\beta}} \bar{y}_{i} g_{i}(\bar{u}) \bar{p}\right\}\right) \leqq-\sigma_{\beta} d^{2}(\bar{x}, \bar{u}),
$$

The sublinearity of $F$ and (4.16) with $\alpha(\bar{x}, \bar{u})>0$ imply 


$$
\begin{aligned}
& F\left(\bar{x}, \bar{u} ; \nabla \sum_{i \in M \backslash I_{0}} \bar{y}_{i} g_{i}(\bar{u})+\nabla^{2} \sum_{i \in M \backslash I_{0}} \bar{y}_{i} g_{i}(\bar{u}) \bar{p}\right) \\
& \leqq \sum_{\beta=1}^{r} F\left(\bar{x}, \bar{u} ; \nabla \sum_{i \in I_{\beta}} \bar{y}_{i} g_{i}(\bar{u})+\nabla^{2} \sum_{i \in I_{\beta}} \bar{y}_{i} g_{i}(\bar{u}) \bar{p}\right) \\
& \leqq-\frac{1}{\alpha(\bar{x}, \bar{u})}\left(\sum_{\beta=1}^{r} \sigma_{\beta}\right) d^{2}(\bar{x}, \bar{u}) .
\end{aligned}
$$

The first dual constraint and the above inequality along with the sublinearity of $F$ imply

$$
\begin{aligned}
& F\left(\bar{x}, \bar{u} ; \nabla \bar{\lambda}^{t} f(\bar{u})+\nabla^{2} \bar{\lambda}^{t} f(\bar{u}) \bar{p}+\nabla \sum_{i \in I_{0}} \bar{y}_{i} g_{i}(\bar{u})+\nabla^{2} \sum_{i \in I_{0}} \bar{y}_{i} g_{i}(\bar{u}) \bar{p}\right) \\
& \geqq \frac{1}{\alpha(\bar{x}, \bar{u})}\left(\sum_{\beta=1}^{r} \sigma_{\beta}\right) d^{2}(\bar{x}, \bar{u}) .
\end{aligned}
$$

Since

$$
\left[\frac{1}{\alpha(\bar{x}, \bar{u})} \sum_{\beta=1}^{r} \sigma_{\beta}+\frac{\rho}{\alpha_{1}(\bar{x}, \bar{u})}\right] \geqq 0
$$

we have

$$
\begin{aligned}
& F\left(\bar{x}, \bar{u} ; \nabla \bar{\lambda}^{t} f(\bar{u})+\nabla^{2} \bar{\lambda}^{t} f(\bar{u}) \bar{p}+\nabla \sum_{i \in I_{0}} \bar{y}_{i} g_{i}(\bar{u})+\nabla^{2} \sum_{i \in I_{0}} \bar{y}_{i} g_{i}(\bar{u}) \bar{p}\right) \\
& \geqq-\frac{\rho}{\alpha_{1}(\bar{x}, \bar{u})} d^{2}(\bar{x}, \bar{u}) .
\end{aligned}
$$

Since $\alpha_{1}(\bar{x}, \bar{u})>0$, we obtain

$$
\begin{aligned}
& F\left(\bar{x}, \bar{u} ; \alpha_{1}(\bar{x}, \bar{u})\left\{\nabla \bar{\lambda}^{t} f(\bar{u})+\nabla^{2} \bar{\lambda}^{t} f(\bar{u}) \bar{p}+\nabla \sum_{i \in I_{0}} \bar{y}_{i} g_{i}(\bar{u})+!+\nabla^{2} \sum_{i \in I_{0}} \bar{y}_{i} g_{i}(\bar{u}) \bar{p}\right\}\right) \\
& \geqq-\rho d^{2}(\bar{x}, \bar{u}),
\end{aligned}
$$

which by the strict second order $\left(F, \alpha_{1}, \rho, d\right)$-pseudoconvexity of $\bar{\lambda}^{t} f+\sum_{i \in I_{0}} \bar{y}_{i} g_{i}$ at $\bar{u}$ yields

$$
\bar{\lambda}^{t} f(\bar{x})+\sum_{i \in I_{0}} \bar{y}_{i} g_{i}(\bar{x})>\bar{\lambda}^{t} f(\bar{u})+\sum_{i \in I_{0}} \bar{y}_{i} g_{i}(\bar{u})-\frac{1}{2} \bar{p}^{t} \nabla^{2}\left[\bar{\lambda}^{t} f(\bar{u})+\sum_{i \in I_{0}} \bar{y}_{i} g_{i}(\bar{u})\right] \bar{p} .
$$

The above inequality in view of $\sum_{i \in I_{0}} \bar{y}_{i} g_{i}(\bar{x}) \leqq 0$ contradicts (4.14). 
On the other hand, when hypothesis (ii) holds, the strict second order $\left(F, \alpha, \sigma_{\beta}, d\right)$-pseudoconvexity of $\sum_{i \in I_{\beta}} \bar{y}_{i} g_{i}$ and (4.15) yield

$$
F\left(\bar{x}, \bar{u} ; \alpha(\bar{x}, \bar{u})\left\{\nabla \sum_{i \in I_{\beta}} \bar{y}_{i} g_{i}(\bar{u})+\nabla^{2} \sum_{i \in I_{\beta}} \bar{y}_{i} g_{i}(\bar{u}) \bar{p}\right\}\right)<-\sigma_{\beta} d^{2}(\bar{x}, \bar{u}),
$$

Using the sublinearity of $F$ and $\alpha(\bar{x}, \bar{u})>0$, it follows from the above inequality that

$$
\begin{aligned}
& F\left(\bar{x}, \bar{u} ; \nabla \sum_{i \in M \backslash I_{0}} \bar{y}_{i} g_{i}(\bar{u})+\nabla^{2} \sum_{i \in M \backslash I_{0}} \bar{y}_{i} g_{i}(\bar{u}) \bar{p}\right)<-\frac{1}{\alpha(\bar{x}, \bar{u})}\left(\sum_{\beta=1}^{r} \sigma_{\beta}\right) d^{2}(\bar{x}, \bar{u}) \\
& \left.\leqq \frac{\rho}{\alpha_{1}(\bar{x}, \bar{u})}\right) d^{2}(\bar{x}, \bar{u}) .
\end{aligned}
$$

Therefore, from the first dual constraint and the sublinearity of $F$ with $\alpha_{1}(\bar{x}, \bar{u})>0$, we have

$$
\begin{aligned}
& F\left(\bar{x}, \bar{u} ; \alpha_{1}(\bar{x}, \bar{u})\left\{\nabla \bar{\lambda}^{t} f(\bar{u})+\nabla^{2} \bar{\lambda}^{t} f(\bar{u})+\nabla \sum_{i \in I_{0}} \bar{y}_{i} g_{i}(\bar{u})+\nabla^{2} \sum_{i \in I_{0}} \bar{y}_{i} g_{i}(\bar{u}) \bar{p}\right\}\right) \\
& >-\rho d^{2}(\bar{x}, \bar{u}),
\end{aligned}
$$

which by the virtue of second order $\left(F, \alpha_{1}, \rho, d\right)$-quasiconvexity of $\bar{\lambda}^{t} f+\sum_{i \in I_{0}} \bar{y}_{i} g_{i}$ at $\bar{u}$ becomes

$\bar{\lambda}^{t} f(\bar{x})+\sum_{i \in I_{0}} \bar{y}_{i} g_{i}(\bar{x})>\bar{\lambda}^{t} f(\bar{u})+\sum_{i \in I_{0}} \bar{y}_{i} g_{i}(\bar{u})-\frac{1}{2} \bar{p}^{t} \nabla^{2}\left[\bar{\lambda}^{t} f(\bar{u})+\sum_{i \in I_{0}} \bar{y}_{i} g_{i}(\bar{u})\right] \bar{p}$.

Since $\bar{x}$ is feasible for (MP) and $\bar{y} \geqq 0$, we have

$$
\bar{\lambda}^{t} f(\bar{x})>\bar{\lambda}^{t} f(\bar{u})+\sum_{i \in I_{0}} \bar{y}_{i} g_{i}(\bar{u})-\frac{1}{2} \bar{p}^{t} \nabla^{2}\left[\bar{\lambda}^{t} f(\bar{u})+\sum_{i \in I_{0}} \bar{y}_{i} g_{i}(\bar{u})\right] \bar{p},
$$

again contradicting (4.14). Hence, in both cases $\bar{x}=\bar{u}$.

Acknowledgment. The authors express their deep gratitude to the anonymous referee for his/her valuable suggestions which have improved the presentation of the paper. 


\section{References}

[1] Aghezzaf, B., Hachimi, M., Sufficiency and duality in multiobjective programming involving generalized $(F, \rho)$-convexity, J. Math. Anal. Appl. 258 (2001), 617-628.

[2] Ahmad, I., Sufficiency and duality in multiobjective programming with generalized $(F, \rho)$-convexity, J. Appl. Anal. 11 (2005), 19-33.

[3] Ahmad, I., Husain, Z., Second order $(F, \alpha, \rho, d)$-convexity and duality in multiobjective programming, Inform. Sci. 176 (2006), 3094-3103.

[4] Ahmad, I., Husain, Z., Optimality conditions and duality in nondifferentiable minimax fractional programming with generalized convexity, J. Optim. Theory Appl. 129 (2006), 255-275.

[5] Ahmad, I., Husain, Z., Duality in nondifferentiable minimax fractional programming with generalized convexity, Appl. Math. Comput. 176 (2006), 545-551.

[6] Chinchuluun, A., Pardalos, P. M., Multiobjective programming problems under generalized convexity, in: "Models and Algorithms for Global Optimization", Springer Optim. Appl. 4, Springer, New York, 2007, 3-20.

[7] Gulati, T. R., Ahmad, I., Agarwal, D., Sufficiency and duality in multiobjective programming under generalized type I functions, J. Optim. Theory Appl. 135 (2007), 411-427.

[8] Gulati, T. R., Islam, M. A., Sufficiency and duality in multiobjective programming involving generalized F-convex functions, J. Math. Anal. Appl. 183 (1994), 181-195.

[9] Hanson, M. A., Mond, B., Further generalizations of convexity in mathematical programming, J. Inform. Optim. Sci. 3 (1982), 25-32.

[10] Liang, Z. A., Huang, H. X., Pardalos, P. M., Optimality conditions and duality for a class of nonlinear fractional programming problems, J. Optim. Theory Appl. 110 (2001), 611-619.

[11] Liang, Z. A., Huang, H. X., Pardalos, P. M., Efficiency conditions and duality for a class of multiobjective fractional programming problems, J. Global Optim. 27 (2003), 447-471.

[12] Maeda, T., Constraint qualifications in multiobjective optimization problems: Differentiable case, J. Optim. Theory Appl. 80 (1994), 483-500.

[13] Mangasarian, O.L., Second and higher order duality in nonlinear programming, J. Math. Anal. Appl. 51 (1975), 607-620.

[14] Mond, B., Second order duality for nonlinear programs, Opsearch 11 (1974), 90-99.

[15] Mond, B., Zhang, J., Duality for multiobjective programming involving second order $V$-invex functions, in: "Proceedings of the Optimization Miniconference", B. M. Glower and V. Jeyakumar (eds.), University of Ballarat, Ballarat (Australia), 1995, 89-100.

[16] Preda, V., On efficiency and duality for multiobjective programs, J. Math. Anal. Appl. 166 (1992), 365-377.

[17] Vial, J. P., Strong and weak convexity of sets and functions, Math. Oper. Res. 8 (1983), 231-259.

[18] Yuan, D. H., Chinchuluun, A., Liu, X. L., Pardalos, P. M., Optimality conditions and duality for multiobjective programming involving $(C, \alpha, \rho, d)$-type I functions, in: "Generalized Convexity and Generalized Monotonicity", Lecture Notes in Econom. and Math. Systems 583, Springer, Berlin, 2007, 73-87. 
[19] Zhang, J., Mond, B., Second order duality for multiobjective nonlinear programming involving generalized convexity, in: "Proceedings of the Optimization Miniconference III", B. M. Glower, B.D. Craven and D. Ralph (eds.), University of Ballarat, Ballarat (Australia), 1997, 79-95.

IZHAR AHMAD

Department of Mathematics

Aligarh Muslim University

Aligarh-202 002, India

E-MAIL: IZHARMATHS@HOTMAIL.COM
ZAKIR HUSAIN

Department of Mathematics

Aligarh Muslim University

Aligarh-202 002, India

E-MAIL: ZHUSAIN_AMU@HOTMAIL.COM 